\title{
Effect of surfactants on the performance of tubular and spherical micromotors - a comparative study $\dagger$
}

Cite this: RSC Adv., 2014, 4, 20334

\author{
Juliane Simmchen,,$^{\mathrm{abc}}$ Veronika Magdanz, $\neq^{\mathrm{c}}$ Samuel Sanchez, ${ }^{* c d}$ \\ Sarocha Chokmaviroj, ${ }^{\text {ce }}$ Daniel Ruiz-Molina, ${ }^{a b}$ Alejandro Baeza ${ }^{f g}$ \\ and Oliver G. Schmidt ${ }^{\text {ch }}$
}

The development of artificial micromotors is one of the greatest challenges of modern nanotechnology. Even though many kinds of motors have been published in recent times, systematic studies on the influence of components of the fuel solution are widely missing. Therefore, the autonomous movement of Pt-microtubes and Pt-covered silica particles is comparatively observed in the presence and absence of surfactants in the medium. One representative of each of the three main surfactant classes - anionic (sodium dodecyl sulfate, SDS), cationic (benzalkonium chloride, BACl) and non-ionic (Triton X) - has been chosen and studied.

Received 13th March 2014

Accepted 12th April 2014

DOI: $10.1039 / c 4 r a 02202 e$

www.rsc.org/advances

presence of sodium dodecyl sulfate (SDS), an anionic surfac-

\section{Introduction}

Since Whitesides' ground-breaking article on autonomous movement in 2002, nano- and micromotors have turned into a rapidly developing field of science. ${ }^{1}$ Even if there are plenty of types of artificial motors such as nanorods, ${ }^{2-4}$ rotors $^{5}$ and helical structures $^{6-8}$ a large number of publications deals either with microtube ${ }^{9-11}$ or microparticle ${ }^{12-17}$ based micromotors. Especially for the tube morphology, first potential applications of the micromotors approach have recently been presented ${ }^{\mathbf{1 1 , 1 8 - 2 0}}$ while the field of particle motion received several theoretical contributions. $^{21,22}$

Catalytic micromotors are affected by the composition ${ }^{23}$ and temperature of the fuel solution where they swim..$^{\mathbf{2 0 2 4 - 2 6}}$ Very recently Pumera's group analysed the influence of some surfactants on the motion of bubble-propelled tubular microjets. ${ }^{27}$ They observed an enhanced motion of microjets in the

${ }^{a}$ ICN2 - Institut Catala de Nanociencia i Nanotecnologia, Campus UAB, 08193 Bellaterra, Barcelona, Spain

${ }^{b}$ CSIC - Consejo Superior de Investigaciones Cientificas, ICN2 Building Campus UAB, 08193 Bellaterra, Barcelona, Spain

'IFW Dresden, Institute for Integrative Nanosciences, Helmholtzstr 20, 01069 Dresden, Germany

${ }^{d}$ Max-Planck Institute for Intelligent Systems, Heisenbergstr. 3, 70569 Stuttgart, Germany.E-mail: sanchez@is.mpg.de

${ }^{e}$ Faculty of Engineering Chulalonkorn University, Bangkok, Thailand

${ }^{f}$ Dep. de Química Inorgánica y Bioinorgánica, UCM, Plaza Ramón y Cajal s/n, Madrid, Spain

${ }^{g}$ Center on Bioengineering, Biomaterials and Nanomedicine (CIBER-BBN), Spain

${ }^{h}$ Material Systems for Nanoelectronics, Chemnitz University of Technology, Reichenhainerstr.70, 09107 Chemnitz, Germany

$\dagger$ Electronic supplementary information (ESI) available. See DOI: 10.1039/c4ra02202e

\$ Both authors contributed equally to this work. tant. The authors claimed that Tween 20, a non-ionic surfactant with a large molecular mass (four times the mass of the other surfactants employed) slows down the microjets drastically. Their catalytic microjets were not active in cetrimonium bromide (CTAB), a cationic surfactant containing an ammonium moiety with high affinity to platinum, which may be the reason of their surprising results since other cationic surfactants have been previously used in the literature. ${ }^{28}$ Altogether these publications hold a lot of technical expertise on each single system but this knowledge is not necessarily transferable from one kind of motor to another and methodical comparisons are missing in this field.

This manuscript systematically studies two different types of micromotors: Pt-silica Janus particles and microtubes with an inner Pt layer, both self-propelled by the decomposition of the $\mathrm{H}_{2} \mathrm{O}_{2}$ employed as fuel. The main chemical reaction takes place on the platinum components of both types of motors that work as catalyst for degradation of $\mathrm{H}_{2} \mathrm{O}_{2}$. Despite these similarities, different motor morphologies lead to quite different types of dynamics at the microscale. Catalytic microtubes produce bubbles that propel them through a jet-like mechanism. ${ }^{\mathbf{9}, 10}$ It has been claimed that the use of surfactant is necessary to reduce the surface tension and stabilize the generated bubbles ${ }^{\mathbf{1 9 2 9}}$ and for that, different surfactants have been "randomly" used by different groups, partially reporting controversial results for the same surfactant. ${ }^{30}$ In most of the spherical micromotors reported so far, no bubbles were observed, i.e. the jet-like mechanism could not be assumed to be responsible for spherical particle propulsion. Nonetheless, a recent report from Zhao's group showed bubble-driven motion of bigger spherical micromotors. ${ }^{30,31}$ Further investigations towards elucidating the mechanism of motion of spherical Janus particle motors are 
expected in the near future, which is however out of the scope of this paper. Nonetheless, the presented data might be seen as an important experimental base for such considerations. Up to our knowledge, there is no systematic study on the nature and concentration of surfactants required for optimal bubble- and non-bubble-driven self-propulsion.

Here, we analyzed the motion of different types of motors in peroxide solutions without surfactant, noticing that both systems are able to self-propel in $\mathrm{H}_{2} \mathrm{O}_{2}$. A comparative study is given using the relationship speed per catalytic area. We observed different dynamic behaviour at different concentrations of peroxide, i.e. Janus particles self-propel relatively faster at low concentrations but saturate at high concentrations where tubular microjets acquire maximal speeds. ${ }^{26}$ However, microjets are unable to self-propel at low concentrations of peroxide if surfactants are not used. Aiming at finding the optimal conditions of the fuel (peroxide and surfactant) to propel different micromotors, we studied the effect of different types of surfactants in the fuel solution. The three (cationic, anionic, nonionic) surfactants were particularly selected in order to diminish specific interaction as metal-specific absorption. Even though we used a quaternary ammonium salt as positive surfactant, this group is sterically hindered by a long alkylic chain. At the same time we chose surfactants with relatively similar properties considering molecular mass and functional groups.

\section{Experimental section}

\section{Fabrication of catalytic Pt-covered microparticles}

A suspension of spherical silica colloids (diameter of $4.78 \mu \mathrm{m}$, Bangs Laboratories) is dripped onto an oxygen-plasma cleaned glass substrate, followed by slow evaporation of the solvent under ambient conditions. Afterwards, the particle array samples are introduced to the vacuum chamber (base pressure of $1 \times 10^{-7}$ mbar) of a sputtering machine where deposition of the magnetic multilayer stack consisting of $[\mathrm{Co}(0.4 \mathrm{~nm}) /$ $\mathrm{Pt}(0.6 \mathrm{~nm})]_{5}$ is carried out at room temperature (Ar sputter pressure, $8 \times 10^{3}$ mbar) as described by Baraban et al. ${ }^{\mathbf{1 4 5}}$ and a final $5 \mathrm{~nm}$ Pt layer was sputtered to guarantee catalytic properties.

\section{Fabrication of catalytic platinum microtubes}

The fabrication method has been described previously., ${ }^{\mathbf{9} 10}$ In brief, $\mathrm{Ti} / \mathrm{Cr} / \mathrm{Fe} / \mathrm{Pt}$ microjets were fabricated by e-beam deposition of metallic layers onto lithographically patterned photoresist layers. Square $50 \mu \mathrm{m}$ photoresist patterns were prepared on $28 \mathrm{~mm}$ square glass slides. Photoresist AR-P 3510 was spincoated onto the cleaned glass wafers at $3500 \mathrm{rpm}$ for $35 \mathrm{~s}$, followed by a soft bake using a hotplate at $90{ }^{\circ} \mathrm{C}$ for $2 \mathrm{~min}$ and exposure to UV light with a Karl Suss MA56 Mask Aligner (410$605 \mathrm{~nm}$ ) for 7 seconds. Patterns were developed in a 1:1 AR300-35 : $\mathrm{H}_{2} \mathrm{O}$ solution. On-chip rolled-up catalytic microtubes were obtained by a tilted deposition of $5 \mathrm{~nm}$ of each metal ( $\mathrm{Ti}, \mathrm{Fe}, \mathrm{Cr})$ at a $75^{\circ}$ angle. Subsequently, a $1 \mathrm{~nm}$ layer of platinum was sputtered on the glass substrates. By dissolving the photoresist layer in dimethyl sulfoxide, pre-stressed multilayers automatically roll up into microtubes. The supercritical point dryer was adopted in the fabrication of rolled-up microtubes to avoid the tubes collapsing during drying. The diameter of tubes was tuned to be $5 \mu \mathrm{m}$ (comparable to particle size). As a consequence tubes had approximately 3 windings. In order to self-propel catalytic microjets, aqueous hydrogen peroxide solutions with a volume concentration of $5 \%$ were used as chemical fuels, in which certain concentrations of surfactant such as benzalkonium chloride (FlukaChemika), sodium dodecyl sulfate (Sigma-Aldrich), Triton X (SigmaAldrich) or FIT as common dish soap were added to reduce the surface tension.

\section{Speed experiments and evaluation}

Experiments on microtubes were performed in glass recipients containing $\mathrm{H}_{2} \mathrm{O}_{2}$ solutions of different concentrations in distilled water. A glass substrate bearing tubes was put into the solution and the tubes detached from the surface due to forces generated by bubbles.

All microparticle experiments were performed in a 96 well plate in distilled water containing defined concentrations of $\mathrm{H}_{2} \mathrm{O}_{2}$ to which $1 \mu \mathrm{l}$ particles dispersed in distilled water were added. Videos were recorded with a Phantom Miro eX2 high speed camera mounted to an inverted Zeiss AxioVision microscope. Evaluation was performed with the Phantom camera software. Considered videos were at least 4 s long (corresponding to min 82 frames to guarantee high quality videos) Particles could not be followed over a longer distance than one screen width which corresponds to $(0.4365 \mathrm{~mm} ; 512 \times 384$ Pixel) at a magnification of $100 \times$.

\section{Effects of soap on particle-/tube-movement}

As synthesized particles or tubes were added to solutions of different soap concentrations (range from $0.00001 \mathrm{wt} \%$ to $10 \mathrm{wt} \%$ ), containing $5 \%$ of $\mathrm{H}_{2} \mathrm{O}_{2}$. Using a Phantom camera videos were recorded with 20 frames per second, with a minimum size of 82 frames, speed evaluation was performed using the integrated mode of the Phantom software.

\section{Zetapotential measurement}

Due to methodical limitations original Janus particles could not be used to evaluate particle charge (zetapotential) relative to tenside concentrations. $10 \mathrm{~nm} \mathrm{Au}$ and Pt were therefore evaporated on $1 \mu \mathrm{m}$ silica particles and measured in bidistilled water containing determined surfactant concentrations. Zetapotential was measured in Malvern one-way Zetapotential cells. From the measurements it can be concluded, that surfactants that have strong effects on the behaviour of the Janus particle motors also strongly change the surface potential. The critical micelle concentration (SDS: $0.23 \mathrm{wt} \%$, Triton X: $1.375 \times 10^{-2} \mathrm{wt} \%$, BACl $0.205 \mathrm{wt} \%$ ) does not seem to have an important impact on the surface potential. This is an indication that the coverage of $\mathrm{Pt}$ with surfactant molecules only plays a minor part in the mechanism. 


\section{Results and discussion}

First we aim to characterize the motion of both kinds of catalytic motors in pure diluted peroxide and compare this later with tenside containing solutions. Fig. 1 displays the speed of tubes and particles both in absolute values and relative to their catalytic area as a function of $\mathrm{H}_{2} \mathrm{O}_{2}$ concentration without any surfactant. The absolute values of particle speed are generally lower than tube velocities (Fig. 1a) and the speed behaviour for particles is in quite good accordance with data published previously by Howse et al. ${ }^{14,17}$

It is known that the speed of microtubular motors depends on the fuel concentration and generally increases with higher hydrogen peroxide concentration. As shown in Fig. 1, in a surfactant-free hydrogen peroxide solution the microjets do not show any movement at concentrations lower than $5 \% \mathrm{H}_{2} \mathrm{O}_{2}$. At 2 and $5 \%$ of peroxide some microjets start producing bubbles (see Table 1) but nonetheless do not lead to movement of the tubes. Oxygen bubble generation depends on the concentration of hydrogen peroxide and the catalytic rate of decomposition of $\mathrm{H}_{2} \mathrm{O}_{2}$ into oxygen and water. Therefore, at concentrations of 2 and $5 \% \mathrm{H}_{2} \mathrm{O}_{2}$, it is possible to generate enough oxygen inside the cavity of the tubes that accumulates into visible bubbles. However, without surfactant in the fuel solution, the surface tension is too large for the bubbles to freely and continuously
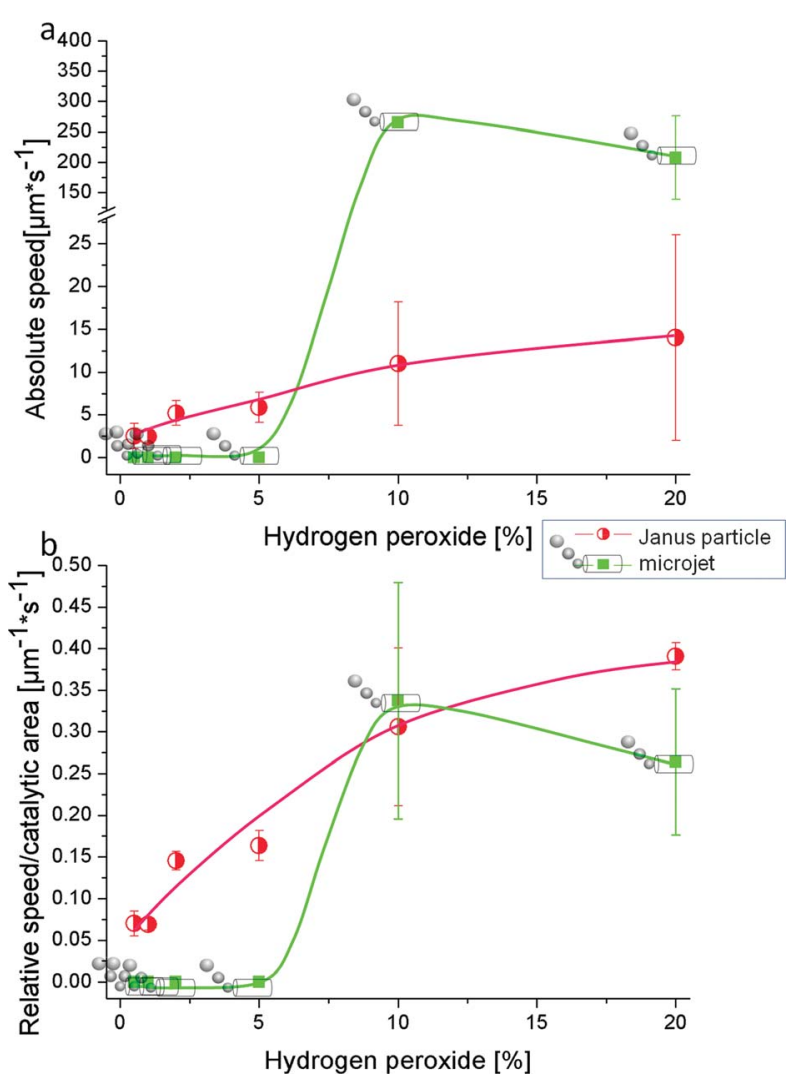

Fig. 1 Comparative speeds of Janus micromotors and bubbling microtubular jets in peroxide fuel in the absence of surfactant. (a) Absolute speed and (b) relative speed per catalytic Pt area over hydrogen peroxide concentration.
Table 1 Hydrogen peroxide concentration (wt\%) and the corresponding percentage of microtubes producing bubbles, * marked items produced bubbles but did not move

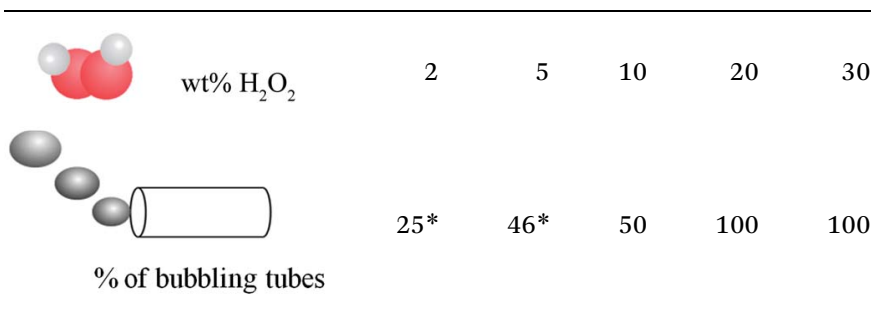

exit the tube. To achieve motion at low Reynolds numbers, generation forces must be continuously generated since viscous forces govern at the nanoscale. ${ }^{32}$

It has been also reported that upon increase in surface tension in the fuel solution, the bubbles are pinned to the opening of the tube which leads to microjets which cannot propel forward. ${ }^{33}$

We surprisingly observed a few tubes moving without bubbles at very low speeds (approx. $60 \mu \mathrm{m} \mathrm{s}^{-1}$ ) compared with bubbling tubes (see ESI 1 and $2 \dagger$ ). These results demonstrate that microjets can move without surfactant, what made us suspect that two different mechanisms (phoretic and bubble propelled mechanism) may coexist depending on the fuel conditions, similar to what Wilson et al. described for stomatocytes. ${ }^{34}$

It is accepted that in absence of surfactants the bubbles tend to be larger than with surface tension decreasing agents and less frequent, which seems not to be the optimal case for motion at low Reynolds numbers. ${ }^{26,32}$ At higher hydrogen peroxide percentages the local concentration of $\mathrm{O}_{2}$ is high enough to overcome surface tension and form bubbles that continuously propel the tubes. Janus particles also increase their speed with increasing fuel concentration (see Videos ESI 3 and $4 \dagger$ ), but only up to a threshold similar to the Michaelis-Menten-like limit for enzymes. ${ }^{17}$ Although the absolute values Janus particles reach are much lower than those of microjets (Fig. 1a), the trend of relative speed per catalytic area totally changes. At low concentrations of $\mathrm{H}_{2} \mathrm{O}_{2}$ Janus particles present higher relative speed, whereas bubble propelled microtubes only start to self-propel at $10 \%$ peroxide, where coincidentally similar relative values are obtained for both types of micromotors. Fig $1 \mathrm{~b}$ indicates that at low concentrations of peroxide and surfactant-free conditions, the mechanism of motion is more efficient per catalytic area for Janus micromotors than for tubular jets. As our particles do not produce any visible bubbles and the production of nano-sized bubbles is highly unlikely as a very high surface tension would have to be overcome, the mechanism for particle movement cannot be described as bubble propulsion. Currently, the most accepted theory to explain particle motion is the ratio of reactants to products $(2: 3)$, which produces a difference in entropy around the particle, leading to a pressure difference impelling the particle, i.e. diffusiophoresis. ${ }^{35-37}$ A related assumption is the occurrence of a driving force due to a localized gradient of oxygen molecules (leading equally to self-diffusiophoresis) and recent papers indicate that some electrokinetic mechanism may also be 
present. $^{21,22}$ In contrast to spherical particles, propulsion of Pttubes has been considered doubtlessly due to bubble formation.

Mallouk et al. reported the evaluation of micromotor performances by comparing the mechanical power output of a given micromotor with its total chemical power input and identified some sources of energy loss, especially for electrophoretically moving nanorods. ${ }^{38}$ Their comparison with other motor types such as catalytic microtubes and helical magnetical motors lead to the conclusion that both are more efficient than self-electrophoretic motors. Considering relative speed values their affirmation that bubble propulsion is more efficient than phoresisbased mechanisms could not be confirmed in our comparative work when only $\mathrm{H}_{2} \mathrm{O}_{2}$ is employed as fuel component. ${ }^{38}$

Since a very different behaviour of Pt-microjets and Pt Janus particles in diluted hydrogen peroxide solutions are presented, we aimed to increase microjet's speed at low peroxide concentrations and therefore we compared their behaviour in presence of several kinds of surfactants. It is known that the speed of microjets depends on the concentration of peroxide fuel, ${ }^{28,39}$ and the addition of surface tension reducing agents improves the bubble formation. Up to our knowledge, most previous publications on microjets use surfactant containing fuel solutions. Recently, Manjare et al. demonstrated the performance of catalytic microparticles ${ }^{30}$ and tubular graphene oxide-Ti-Pt engines $^{31}$ in $5 \% \mathrm{H}_{2} \mathrm{O}_{2}$ without surfactants depending on their geometry. A variety of surfactants have been applied (benzalkonium chloride, ${ }^{29}$ SDS, ${ }^{40}$ the commercial dish soap FIT, ${ }^{26}$ sodium cholate, ${ }^{41}$ isopropanol, ${ }^{23}$ Triton X). ${ }^{16}$ Sanchez et al. described the effects of the surfactants as mediator for capillary fluid filling, stabilizer for bubbles and reductor of surface tension without deeper investigation of the detailed effect of the surfactants on the micromotors. ${ }^{26}$ Wang's group used Triton $\mathrm{X}$ in their studies without providing details on the role of surfactant. Moreover, they showed ${ }^{\mathbf{4 2}}$ that the propulsion of a microcone jet is reduced when surfactant is added, attributing this fact to lower propulsion forces generated by smaller bubbles compared with larger ones when surfactant is not used. Here, four types of tensides were selected, representing different surfactant classes: Triton $\mathrm{X}$ as nonionic, SDS as anionic and BACl as cationic surfactant, as well as FIT as an example of a commercially available mixture of anionic surfactants (dish soap). The experiments presented in Fig. 2 were performed in distilled water containing $5 \% \mathrm{H}_{2} \mathrm{O}_{2}$ and surfactants in a concentration range between $0.0001 \mathrm{wt} \%$ and $10 \mathrm{wt} \%$. The speed of microjets, as displayed in Fig. 2a, increase with higher surfactant concentration following different trends. This phenomenon can be explained by a tension-reducing effect by the surfactant which is necessary to enable bubble formation in tubular structures which rapidly leaves the cavity of the tube.

The addition of surfactant to the peroxide solution affect the speed of Pt-covered Janus particles in an entirely opposite fashion starting at very low concentrations: from surfactant concentrations of only $0.5 \%$ the particle speed does not achieve as high values as without surfactant (Fig. 1). First assumption was the passivation of Pt similar to the passivation by thiols ${ }^{23}$ but the strong motion inhibiting effect of soap on particles might be only partially due to the coverage of the catalytically active Pt.

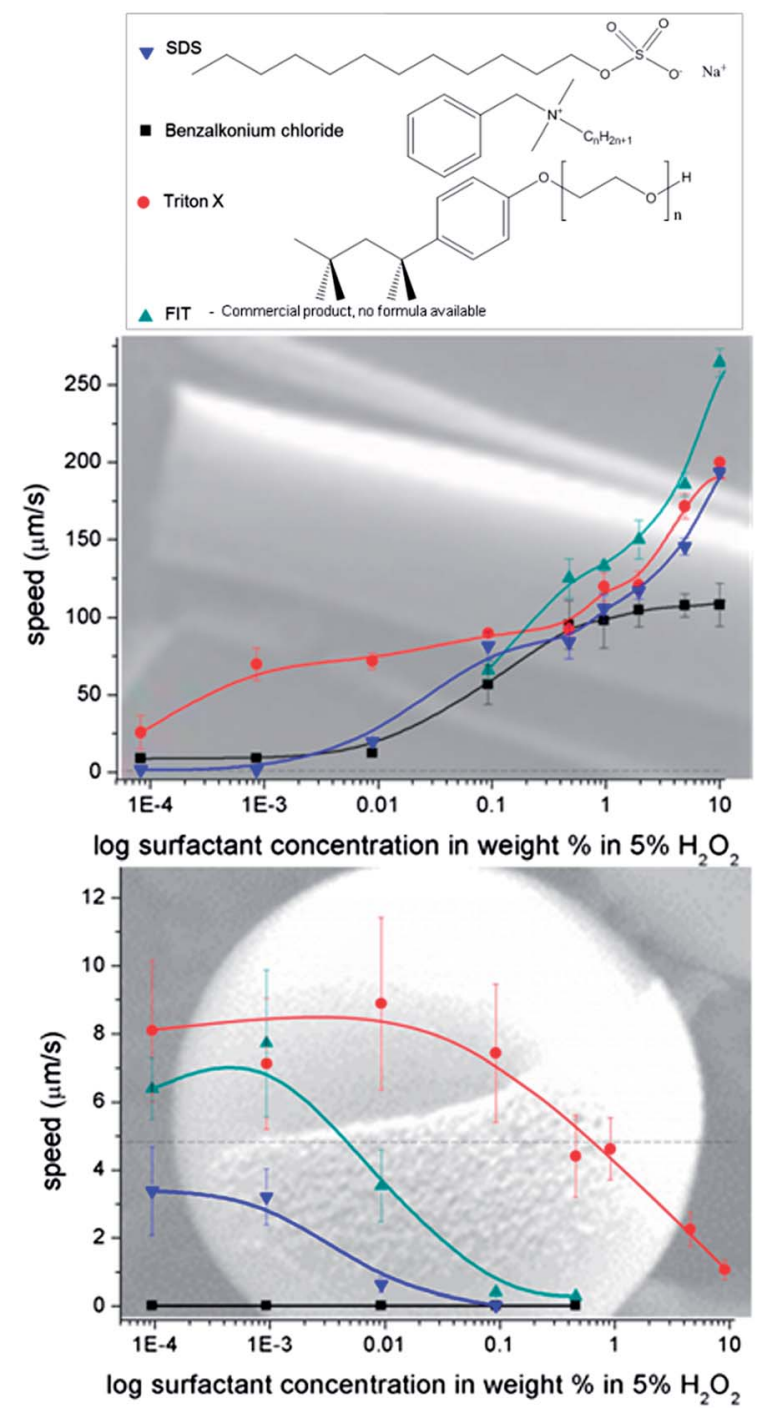

Fig. 2 Effect of surfactant concentration in $5 \% \mathrm{H}_{2} \mathrm{O}_{2}$ solution on average speed of Pt-microtubes (a) and Pt covered microparticles (b); dotted lines depict the maximum speed at surfactant free conditions. Inset: chemical structures of Triton X (nonionic), sodium dodecyl sulfate (anionic), benzalkonium chloride (cationic), FIT (mixture of surfactants).

The critical micelle concentration of these surfactants (SDS: $0.23 \mathrm{wt} \%$, Triton X: $0.22 \mathrm{wt} \%$, benzalkonium chloride $0.205 \mathrm{wt} \%$ (ref. 43)) does not seem to have an important impact neither on the surface potential nor on the speed behaviour. This should be interpreted as a strong indication that a poisoning effect through the coverage of Pt with surfactant molecules only plays a partial role in the mechanism (compare with ref. 23). Another possible reason might lie in the mechanism of motion. The reason for Janus particle movement excludes bubble propulsion since no bubbles are visible. In very recent papers self-electrophoretic mechanisms have been introduced into the discussion about the motion of Janus particles. ${ }^{\mathbf{2 1 , 2 2}}$ But all those results together with our data presented here might lead to the conclusion that multiple mechanisms overlay and are responsible for the resulting motion. The propulsion has to be attributed to a 
phoretic mechanism either caused by oxygen gradients or to ionic gradients caused by the reaction product ratio.

To estimate the impact of surfactant molecules on the ionic properties of the solution we measured the Zetapotential (ZP) of the Janus particles in different surfactant solutions. The measurements confirm that the surfactants have a strong influence on the particles' surface properties as displayed in Fig. 3. Diluted suspensions of Pt-covered particles in deionized water were placed in a disposable cell in an alternating electrical field, generated by the Zetasizer, Malvern Instruments. The electromagnetic field causes electrophoresis and through optical interaction with laser light the mobility of particles is determined, from which the ZP can be calculated. ${ }^{\mathbf{4 4}}$

The initial value of the $\mathrm{ZP}$ at extremely low surfactant concentration coincides with the $\mathrm{ZP}$ value measured in pure water at around $-40 \mathrm{mV}$, which is mainly due to the Silanol groups on the particle surface. This value was compared to non covered silica particle, which showed a slightly more negative potential (data not shown). The effects of the four tensides were very different from each other.

The non-ionic Triton $\mathrm{X}$ did hardly induce any changes in the zetapotential until added concentrations reached 0.01 wt $\%$ when the value shifts towards more neutral values (an expected behaviour at high concentrated solutions caused by compression of the double layer). The motion was almost unaffected at low concentrations and then speed slowly decays. At very high concentrations (1-10\% Triton $\mathrm{X}$ ) the reduction of speed might be due to viscosity changes.

SDS constantly increases the negative $\mathrm{ZP}$ down to $-70 \mathrm{mV}$. Strong inhibition of motion is found using SDS as anionic surfactant, concentrations as low as $0.5 \mathrm{wt} \%$ completely stalled particle motion.

FIT also causes higher absolute $\mathrm{ZP}$ values up to $-65 \mathrm{mV}$. FIT slows particles down at concentrations higher than 0.001 wt $\%$ for FIT as can be observed in video ESI $5 . \dagger$ This behaviour is due to inclusion of the anionic species into the electrochemical double layer (see Fig. 3b).

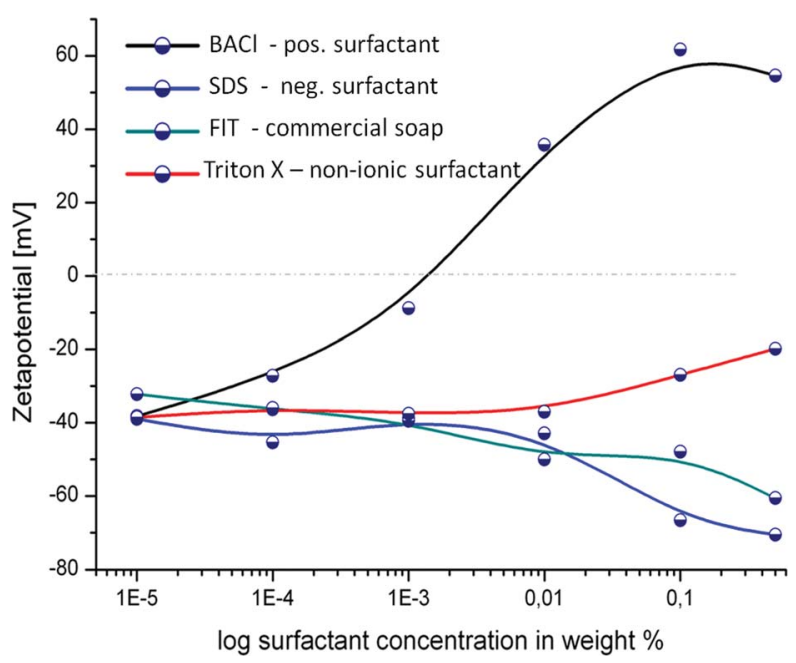

Fig. 3 Effect of surfactant concentration in water on zetapotential of Pt covered silica particles.
The strongest changes can be observed in presence of the cationic surfactant BACl. A concentration of $0.01 \mathrm{wt} \%$ of that surfactant, not only neutralized but inversed the negative zetapotential of the half-covered Pt-particles. At the same time, addition of the cationic surfactant BACl inhibited any movement even at a concentration of only $0.0001 \mathrm{wt} \%$ as can be seen in Fig. 2 and video ESI $6 . \dagger$ Due to the negative surface charge of the silica particles additional electrostatic interactions increase the impact of positive tensides on the double layer (inner and outer Helmholtz-layer, see figure in the $\operatorname{ESI}^{+44}$ ) since they strongly alter the total charge of the particle surface area. As previously described, the results of the $\mathrm{ZP}$ measurements fit well with the speed changes of the Janus particle motors, i.e. those surfactants that strongly affected the speed also provoked significant changes in the absolute zetapotential values and vice versa.

For an explanation we might assume that the particle movement is provoked at least partially by an ion gradient (ionic diffusiophoresis) created by the multistage decomposition of hydrogen peroxide in presence of Pt which involves the formation and decomposition of negative peroxide radicals. ${ }^{45,46}$ This means that the gradient has to be build up in a highly charged medium in case of SDS, FIT and BACl, lowering the relative intensity of the gradient and therefore probably the propulsion force for microparticle movement. Those experiments may help to elucidate this current discussion in the literature about the mechanism of movement of catalytic Janus particles.

As we could observe, the optimal working conditions for both systems differ. While in microtubular jets the presence of a surfactant is essential to reduce surface tension and allow liberation of bubbles, in a spherical particle system the same molecules slow down the movement or even stop it completely. We compared the performance of both motor classes at "optimal" conditions: optimal particle velocities are extracted from experiments in a $5 \% \mathrm{H}_{2} \mathrm{O}_{2}$ solution in pure water. Comparing maximum average speeds (data from Fig. 1a and 2) the used microtubes are almost 20 times faster than particles of a comparable diameter.

We decided to use the concept of "engine output" ${ }^{\mathbf{4 7}}$ or power as probably most comparable parameter, evaluating the approximate work the micro-scale objects perform for their displacement per time unit. As "optimal" tube conditions, we analyzed experiments performed at $5 \% \mathrm{H}_{2} \mathrm{O}_{2}$ solution, containing $5 \%$ surfactant. Those values reveal that in presence of surfactants the microtubular jet is more powerful than particles, using the same catalyst and fuel, while in surfactant free conditions particles perform better. The bubble-propulsion mechanism seems to yield a more efficient drive than propulsion through gradient differences.

Evaluating velocity/aspect ratios Howse et al. ${ }^{17}$ reached the conclusion that spherical geometry is better than rod like micromotors, but as geometries and hydrodynamic effects are not comparable, we see "engine power" as probably more meaningful parameter. This is supported by Mallouk's group's latest work - the evaluation of the motor's "power conversion efficiency" and comparison of this parameter for several kinds of motors. ${ }^{38}$ Their publication concludes that bubble driven 
Table 2 Comparison of shape, weight, catalytic surface, speed and engine power of Pt Janus particles and Pt microtubular jets under optimum surfactant conditions for each case ${ }^{a}$

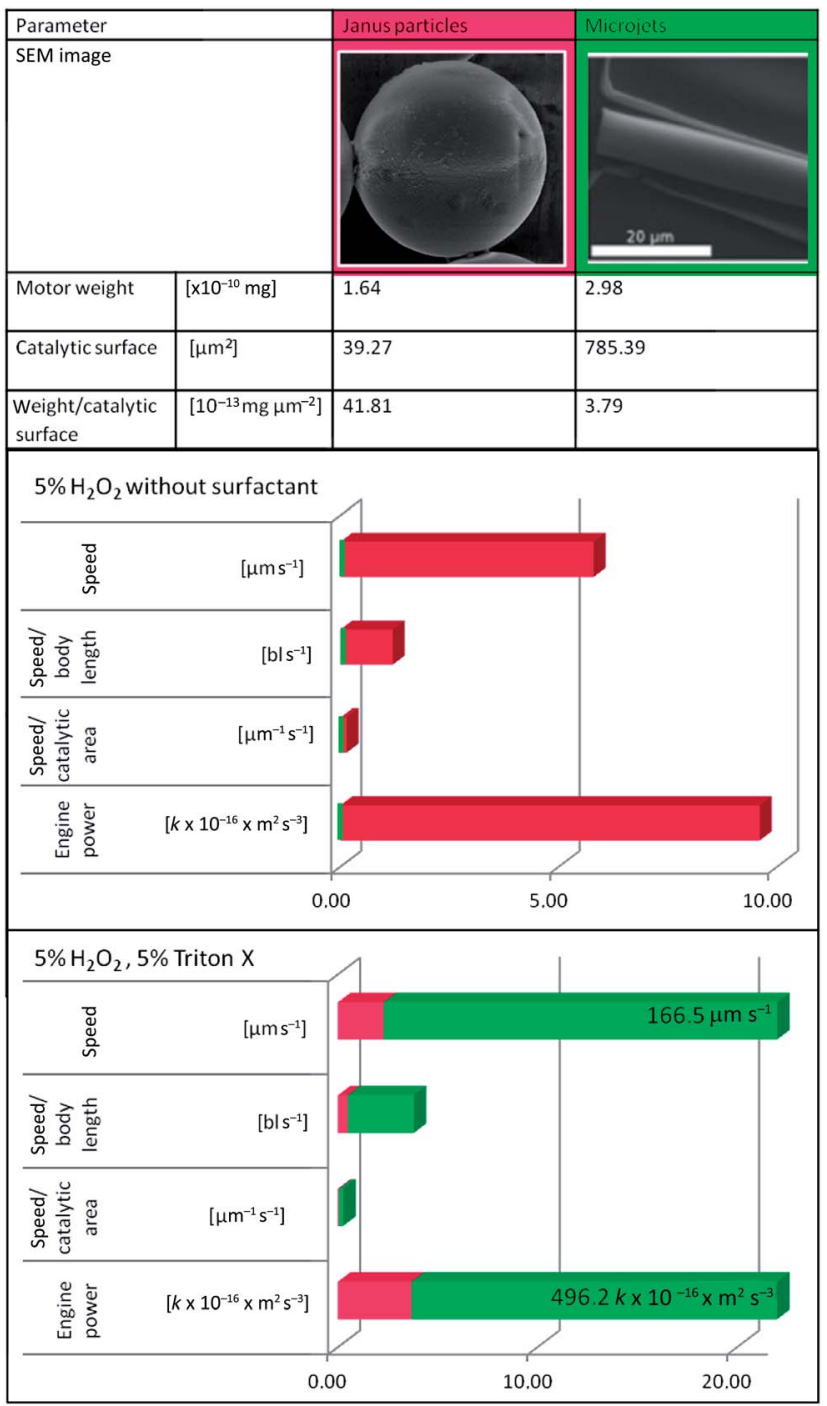

$a$ "Engine power" $=($ force $\times$ way $) /$ time $=$ speed $\times$ weight $\times$ const. in $\left[\mathrm{m} \mathrm{s}^{-2}\right]$ const. corresponds to acceleration.

motors have very low efficiencies, like most of the existing motors do today. To improve the performance of motors, Mallouk and coworkers expose several stages where energy is lost to other phenomena than movement. Assuming that bubble formation is one of those critical points adding surfactant helps the bubble formation and reduces the surface tension assisting the release of bubbles freely from the tubes. As the motion is based on bubble release the higher the frequency of bubbles the higher the speed of motion. ${ }^{\mathbf{1 0}}$

As can be seen in the data shown in Table 2 (green bars) the speed and the engine power of tubes increases drastically after surfactant addition. The opposite conclusion can be drawn for particles. In this case the mechanism is highly probable to be ionic self-diffusiophoresis. The addition leads to less efficient creation of gradients, resulting in a lower power conversion, that is reflected in slower movement and worse "engine power" of microparticles.

\section{Conclusions}

In summary, we characterized the movement of spherical and tubular micromotors in surfactant-free $\mathrm{H}_{2} \mathrm{O}_{2}$ solutions and compared their movements to analogous experiments performed in surfactant containing fuel solution. As examples of micromotors with different motion mechanisms we considered Pt-microjet and Pt covered Janus microparticles. Pt-microjet velocity benefits strongly from the addition of surface tension reducing agents such as SDS, Triton X or benzalkonium chloride to enhance bubble formation in order to propel the tubes, an effect that can be obtained as well by increasing the peroxide percentage. Observation on self propelled non-bubbling tubes in pure diluted peroxide solutions may indicate that various mechanisms could contribute to motion. However this is out of the scope of this paper and further investigations are needed to clarify this issue.

In case of Janus particles the motion and the zetapotentials were shown to be strongly influenced by the different kinds of surfactants. Therefore this study can be seen as evidence of ionic self-diffusiophoresis or as one motion mechanism in Janus microparticles. The here presented results show however that the assumption of one single mechanism for particle propulsion should be revised. To explain the observed phenomena of bubble-free tube movement and the inhibition of Janus particle movement by surfactants the conclusion of overlaid mechanisms is more adequate. Nonetheless, theoretical studies are needed to fully understand those observations.

\section{Acknowledgements}

J.S. thanks R. Nitzsche for an extensive introduction to ZP and the CSIC (Spain) for its predoctoral grant. All authors thank C. Krien for metal deposition and D. Makarov for valuable discussions. S.S. thanks M. Tasinkevych for fruitful discussions. This research was partially supported by grant from Ministerio de Economía y Competitividad; MINECO (grant number MAT2012-38318-C03-02) and the ERC grant "Lab-in-a-tube and Nanorobotics biosensors" (grant number \#311529).

\section{Notes and references}

1 R. F. Ismagilov, A. Schwartz, N. Bowden and G. M. Whitesides, Angew. Chem., Int. Ed., 2002, 41, 652-654. 2 W. F. Paxton, K. C. Kistler, C. C. Olmeda, A. Sen, S. K. St Angelo, Y. Cao, T. E. Mallouk, P. E. Lammert and V. H. Crespi, J. Am. Chem. Soc., 2004, 126, 13424-13431.

3 A. Sen and T. E. Mallouk, Sci. Am., 2009, 72-79.

4 U. K. Demirok, R. Laocharoensuk, K. M. Manesh and J. Wang, Angew. Chem., Int. Ed., 2008, 47, 9349-9351.

5 S. Fournier-Bidoz, A. C. Arsenault, I. Manners and G. A. Ozin, Chem. Commun., 2005, 441-443. 
6 L. Zhang, J. J. Abbott, L. Dong, K. E. Peyer, B. E. Kratochvil, H. Zhang, C. Bergeles and B. J. Nelson, Nano Lett., 2009, 9, 3663-3667.

7 S. Tottori, L. Zhang, F. Qiu, K. K. Krawczyk, A. FrancoObregón and B. J. Nelson, Adv. Mater., 2012, 24, 811-816.

8 A. Ghosh and P. Fischer, Nano Lett., 2009, 9, 2243-2245.

9 Y. Mei, G. Huang, A. A. Solovev, E. B. Ureña, I. Mönch, F. Ding, T. Reindl, R. K. Y. Fu, P. K. Chu and O. G. Schmidt, Adv. Mater., 2008, 20, 4085-4090.

10 A. A. Solovev, Y. Mei, E. Bermúdez Ureña, G. Huang and O. G. Schmidt, Small, 2009, 5, 1688-1692.

11 V. Magdanz, S. Sanchez and O. G. Schmidt, Adv. Mater., 2013, 25, 6581-6588.

12 P. M. Wheat, N. A. Marine, J. L. Moran and J. D. Posner, Langmuir, 2010, 26, 13052-13055.

13 J. Simmchen, A. Baeza, D. Ruiz, M. J. Esplandiu and M. Vallet-Regí, Small, 2012, 8, 2053-2059.

14 L. Baraban, D. Makarov, R. Streubel, I. Mönch, D. Grimm, S. Sanchez and O. G. Schmidt, ACS Nano, 2012, 6, 3383-3389.

15 L. Baraban, M. Tasinkevych, M. N. Popescu, S. Sanchez, S. Dietrich and O. G. Schmidt, Soft Matter, 2012, 8, 48-52.

16 W. Gao, A. Pei and J. Wang, ACS Nano, 2012, 6, 8432-8438.

17 J. R. Howse, R. A. L. Jones, A. J. Ryan, T. Gough, R. Vafabakhsh and R. Golestanian, Phys. Rev. Lett., 2007, 99, 048102.

18 M. Guix, J. Orozco, M. García, W. Gao, S. Sattayasamitsathit, A. Merkoçi, A. Escarpa and J. Wang, ACS Nano, 2012, 6, 44454451.

19 D. Kagan, S. Campuzano, S. Balasubramanian, F. Kuralay, G.-U. Flechsig and J. Wang, Nano Lett., 2011, 11, 2083-2087.

20 L. Soler, V. Magdanz, V. M. Fomin, S. Sanchez and O. G. Schmidt, ACS Nano, 2013, 7, 9611-9620.

21 S. Ebbens, D. A. Gregory, G. Dunderdale, J. R. Howse, Y. Ibrahim, T. B. Liverpool and R. Golestanian, cond-mat. soft, arXiv:1312.6250, 2013.

22 A. T. Brown and W. C. K. Poon, cond-mat. soft, arXiv:1312.4130, 2013.

23 G. Zhao, S. Sanchez, O. G. Schmidt and M. Pumera, Nanoscale, 2013, 5, 2909-2914.

24 L. Soler, C. Martinez-Cisneros, A. Swiersy, S. Sanchez and O. G. Schmidt, Lab Chip, 2012, 13, 4299-4303.

25 S. Balasubramanian, D. Kagan, K. M. Manesh, P. CalvoMarzal, G.-U. Flechsig and J. Wang, Small, 2009, 5, 1569-1574. 26 S. Sanchez, A. N. Ananth, V. M. Fomin, M. Viehrig and O. G. Schmidt, J. Am. Chem. Soc., 2011, 133, 14860-14863.
27 H. Wang, G. Zhao and P. Martin, J. Phys. Chem. C, 2014, 118, 5268-5274.

28 A. A. Solovev, S. Sanchez, M. Pumera, Y. F. Mei and O. G. Schmidt, Adv. Funct. Mater., 2010, 20, 2430-2435.

29 S. Sanchez, A. A. Solovev, S. Schulze and O. G. Schmidt, Chem. Commun., 2011, 47, 698-700.

30 M. Manjare, B. Yang and Y. P. Zhao, Phys. Rev. Lett., 2012, 109, 128305.

31 M. Manjare, B. Yang and Y. P. Zhao, J. Phys. Chem. C, 2013, 117, 4657-4665.

32 E. M. Purcell, Am. J. Phys., 1977, 45, 3-11.

33 A. A. Solovev, E. J. Smith, C. C. Bof ' Bufon, S. Sanchez and O. G. Schmidt, Angew. Chem., Int. Ed., 2011, 50, 10875-10878.

34 D. A. Wilson, B. de Nijs, A. van Blaaderen, R. J. M. Nolte and J. C. M. van Hest, Nanoscale, 2013, 5, 1315-1318.

35 J. L. Anderson, Annu. Rev. Fluid Mech., 1989, 21, 61-99.

36 P. de Buyl and R. Kapral, Nanoscale, 2013, 5, 1337-1344.

37 R. Golestanian, T. B. Liverpool and A. Ajdari, New J. Phys., 2007, 9, 126.

38 W. Wang, T.-Y. Chiang, D. Velegol and T. E. Mallouk, J. Am. Chem. Soc., 2013, 135, 10557-10565.

39 A. A. Solovev, S. Sanchez, Y. Mei and O. G. Schmidt, Phys. Chem. Chem. Phys., 2011, 13, 10131-10135.

40 G. Zhao, S. Sanchez, O. G. Schmidt and M. Pumera, Chem. Commun., 2012, 48, 10090-10092.

41 M. Garcia, J. Orozco, M. Guix, W. Gao, S. Sattayasamitsathit, A. Escarpa, A. Merkoci and J. Wang, Nanoscale, 2013, 5, 1325-1331.

42 K. M. Manesh, M. Cardona, R. Yuan, M. Clark, D. Kagan, S. Balasubramanian and J. Wang, ACS Nano, 2010, 4, 17991804.

43 S. Aldrich, 2013, Product sheets SDS, benzalkonium chloride, Triton X.

44 R. H. Mueller, Zetapotential und Partikelladung in der Laborpraxis - Einführung in die Theorie, praktische Meßdurchführung, Dateninterpretation, Wissenschaftliche Verlagsgesellschaft Stuttgart, 1966.

45 L. Baraban, PhD thesis, University of Konstanz, 2008.

46 H. J. H. Fenton, J. Chem. Soc., Trans., 1894, 65, 899-910.

47 G. B. F.-M. Becker, V. Dietrich, L. Engelmann, C. Ernst, G. Fanghängel, H. Höhne, R. Lenertal, G. Liesenberg, L. Meyer, C. Pews-Hooke, G.-D. Schmidt, A. Stamm and K. Weber, Formeln und Tabellen für die Sekundarstufe I und II, PAETEC Gesellschaft für Bildung und Technik $\mathrm{mbH}$, Berlin, 6th edn, 1996. 